\title{
Aplicação de um modelo de colaboração em um contexto de participação social
}

\author{
Kayque W. R. Oliveira', Melise M. V. Paula', Gustavo E. R. G. Alves ${ }^{1}$, Pedro T. M. \\ Pedrosa $^{2}$, Mauro S. R. Souza ${ }^{2}$, Fabiana Barros ${ }^{2}$ \\ ${ }^{1}$ Instituto de Matemática e Computação - Universidade Federal de Itajubá (UNIFEI) \\ Av. BPS, 1303, Pinheirinho, Itajubá - MG - Brasil \\ ${ }^{2}$ Núcleo Estratégico Interdisciplinar em Resiliência Urbana - Universidade Federal de \\ Itajubá (UNIFEI) \\ Av. BPS, 1303, Pinheirinho, Itajubá - MG - Brasil \\ kayque-willy@hotmail.com, melise@unifei.edu.br, \{gustavo.guedes, \\ pedro.torres, mauro.ribeiro, fabiana.barros\} eneiru.org
}

\begin{abstract}
The objective of this paper is present a study which a collaborative model was used to structure the social participation initiatives in municipal master plan review projects. The study presents a model for organization of participation activities and describes the digital marketing strategies made for communication to publicize the actions taken by management, the mobilization events, the results obtained and the available participation tools. The tools make it easier for citizens to collaborate through discussion of problems, and allow participants to express their opinion to make data collection.
\end{abstract}

Resumo. $O$ objetivo deste artigo é apresentar um estudo no qual um modelo de colaboração foi utilizado para estruturar as iniciativas de participação social em projetos de revisão de planos diretores municipais. No estudo, é apresentado um modelo de organização das atividades de participação, são descritas as estratégias de comunicação realizadas para divulgação das ações tomadas pela gestão e as ferramentas de participação disponíveis. As ferramentas facilitam que o cidadão exerça a colaboração por meio de discussão de problemas e permitem que o participante forneça sua opinião para realizar levantamento de dados.

\section{Introdução}

No contexto da participação cidadã, a gestão pública tem aplicado esforços para envolver a sociedade civil na concepção de políticas públicas, avaliação de serviços e discussões de problemas [Araujo et al. 2011]. Dessa forma, o uso de Tecnologias de Informação e Comunicação (TICs) pode ser uma alternativa para facilitar e motivar a participação social e aproximar o gestor e cidadão [Cortés-Cediel et al. 2018].

A participação da sociedade civil associada ao uso de tecnologias como forma de aproximar o gestor e o cidadão é um fenômeno que se aplica ao contexto de sistemas colaborativos. Uma vez que, os sistemas colaborativos são projetados para permitir que pessoas se comuniquem e trabalhem em conjunto em um espaço compartilhado [Costa et al. 2014]. No caso deste estudo, o trabalho realizado em conjunto é a elaboração de políticas públicas por meio da colaboração entre cidadãos e gestores. 
O objetivo deste estudo é apresentar uma proposta de aplicação de um modelo de colaboração para definir uma estratégia de participação social. Por lei, são diversos os eventos que exigem a participação social. De forma a permitir uma análise mais detalhada da proposta, o contexto considerado será a participação social na revisão de planos diretores. Como fundamentação, no Estatuto da Cidade da Lei n ${ }^{0} 10.257 / 2001$ [Brasil, 2001] é estabelecido a participação social como obrigatória durante processo de revisão de planos diretores.

Esse trabalho está sendo desenvolvido em parceria com o NEIRU (Núcleo Estratégico Interdisciplinar em Resiliência Urbana) que é um núcleo de pesquisa e extensão vinculado a Universidade Federal de Itajubá. O NEIRU atua como centro de desenvolvimento de pesquisas e práticas no tema do planejamento urbano e ambiental. Um dos serviços prestados pelo NEIRU é a revisão de planos diretores municipais. Atualmente, estão sendo executados três projetos cujo objetivo é a revisão de planos diretores de três cidades de pequeno e médio porte no interior do estado de Minas Gerais: Paraguaçu, Cambuí e Pouso Alegre. Dessa forma, toda a problemática discutida no artigo foi definida em função desses projetos.

Em relação à colaboração, foi considerado o modelo de colaboração $4 \mathrm{C}$, que é a união de quatro conceitos: coordenação, comunicação, cooperação e colaboração [Costa et al. 2014]. O modelo 4C é uma adaptação do modelo 3C, no qual é adicionado o conceito de colaboração. Segundo Costa et al. (2014), a coordenação corresponde ao gerenciamento de pessoas, atividades e recursos; a comunicação compreende a troca de mensagens e informações. Para Costa (2014), as tarefas podem ser desenvolvidas em conjunto, por meio da colaboração, ou individualmente, por meio da cooperação. Em ambos os casos, as tarefas executadas tem um objetivo em comum e utilizam um espaço de trabalho compartilhado. Além do modelo $4 \mathrm{C}$, foi utilizado também o modelo de engajamento de Cortés-Cediel et al. (2018).

Uma das contribuições do trabalho é a abordagem definida para estruturar a participação social. Resumidamente, para a comunicação, foram consideradas estratégias de marketing digital com foco na divulgação das ações que estão sendo tomadas pela gestão, resultados obtidos, eventos de mobilização realizados e ferramentas de participação disponíveis. Para a coordenação, todas as atividades relacionadas à mobilização e participação foram estruturadas em um fluxo. Por fim, para a colaboração e cooperação foram utilizadas ferramentas de participação eletrônica com objetivo de facilitar a colaboração dos cidadãos. Uma das ferramentas utilizadas é a rede social SOPA (Sociedade Participativa) de Caetano (2018), que tem como proposta facilitar que o cidadão discuta problemas da sociedade e soluções, tendo como base tecnológica as redes sociais e técnicas de visualização de informação [Caetano, 2018]. A outra ferramenta é o aplicativo Opina Aí, que tem como objetivo coletar opinião dos cidadãos para realizar levantamento de dados.

\section{Modelo de participação social na revisão de planos diretores}

O processo de revisão do plano diretor realizado pelo NEIRU é prioritariamente participativo e sistematizado em quatro fases. A cada fase, são elaborados produtos parciais (documentos) que são validados pela sociedade civil por meio de eventos de mobilização. Esses eventos representam a base da participação e podem ser realizados 
por diferentes mecanismos como, por exemplo, as audiências públicas e a coleta de opinião.

A primeira fase da revisão do plano consiste na estruturação do plano diretor e planejamento da mobilização e comunicação social. Esse planejamento deve ser realizado de forma a garantir o engajamento dos cidadãos nos eventos de participação. $\mathrm{Na}$ segunda fase, o produto elaborado é um diagnóstico da cidade para avaliação de impactos e planejamento de metas para o plano diretor. Esse produto parcial é um exemplo de artefato que deve ser elaborado, obrigatoriamente, de forma participativa. A terceira fase contempla a elaboração de propostas de programas de ação e definição de ferramentas de monitoramento do plano diretor. Por fim, a quarta fase consiste na sistematização do conhecimento acumulado nas fases anteriores para elaboração de normativas técnicas e legislativas que servirão de suporte para gerar a minuta de lei do plano diretor. A minuta da lei posteriormente é proposta pela Prefeitura para ser discutida e votada pela Câmara Municipal. Apesar do caráter técnico abordado nas fases, a participação social é indispensável em todas elas.

Nas seções seguintes, o modelo adotado para garantir a comunicação e a mobilização dos cidadãos será descrito em função dos elementos que compõem o modelo 4C de colaboração.

\subsection{Coordenação}

Conforme o modelo 4C [Costa et al. 2014], na coordenação são organizadas as atividades realizadas na copperação, colaboração e comunicação. Nesse estudo, o objetivo é promover a participação dos cidadãos na revisão de planos diretores por meio de um modelo de colaboração. Dessa forma, as atividades relacionadas à comunicação, cooperação e colaboração devem ser coordenadas de maneira a estimular a participação social.

Para se conseguir participação social, uma discussão também importante é o engajamento dos cidadãos. Engajamento é definido como um estado psicológico permanente que ocorre em vários estágios e intensidades [O’Brien \& Toms, 2008]. No contexto de políticas públicas, o engajamento pode ser entendido como sinônimo de participação política e envolvimento dos cidadãos na tomada de decisões [Cortés-Cediel et al. 2018].

No estudo de Cortés-Cediel et al. (2018) foi proposto um modelo de engajamento no contexto da participação eletrônica, que teve como base o framework de engajamento de O’brien et al. (2008). Para Cortés-Cediel et al. (2018), o engajamento é definido como um ciclo composto por quatro fases: ponto inicial, estado de engajamento, expiração do engajamento e ponto de reengajamento. Segundo CortésCediel et al. (2018), no ponto inicial, a gestão pública estimula o cidadão a participar por meio de divulgação das ações, procedimentos democráticos e as ferramentas de participação disponíveis. Durante o estado de engajamento, os recursos de acessibilidade e usabilidade nas ferramentas de participação elevam o engajamento para um estágio de maturidade. $\mathrm{O}$ engajamento pode então expirar quando a atividade de participação termina, mas pode ser retomado após um período de tempo. Para isso, a gestão pública pode manter os cidadãos informados sobre os resultados parciais das ações e procedimentos democráticos, os resultados das propostas anteriores e as contribuições individuais dos cidadãos para as iniciativas. 
Nesse trabalho, o modelo de Cortés-Cediel et al. (2018) foi utilizado para definir a coordenação das atividades e é ilustrado na Figura 1:

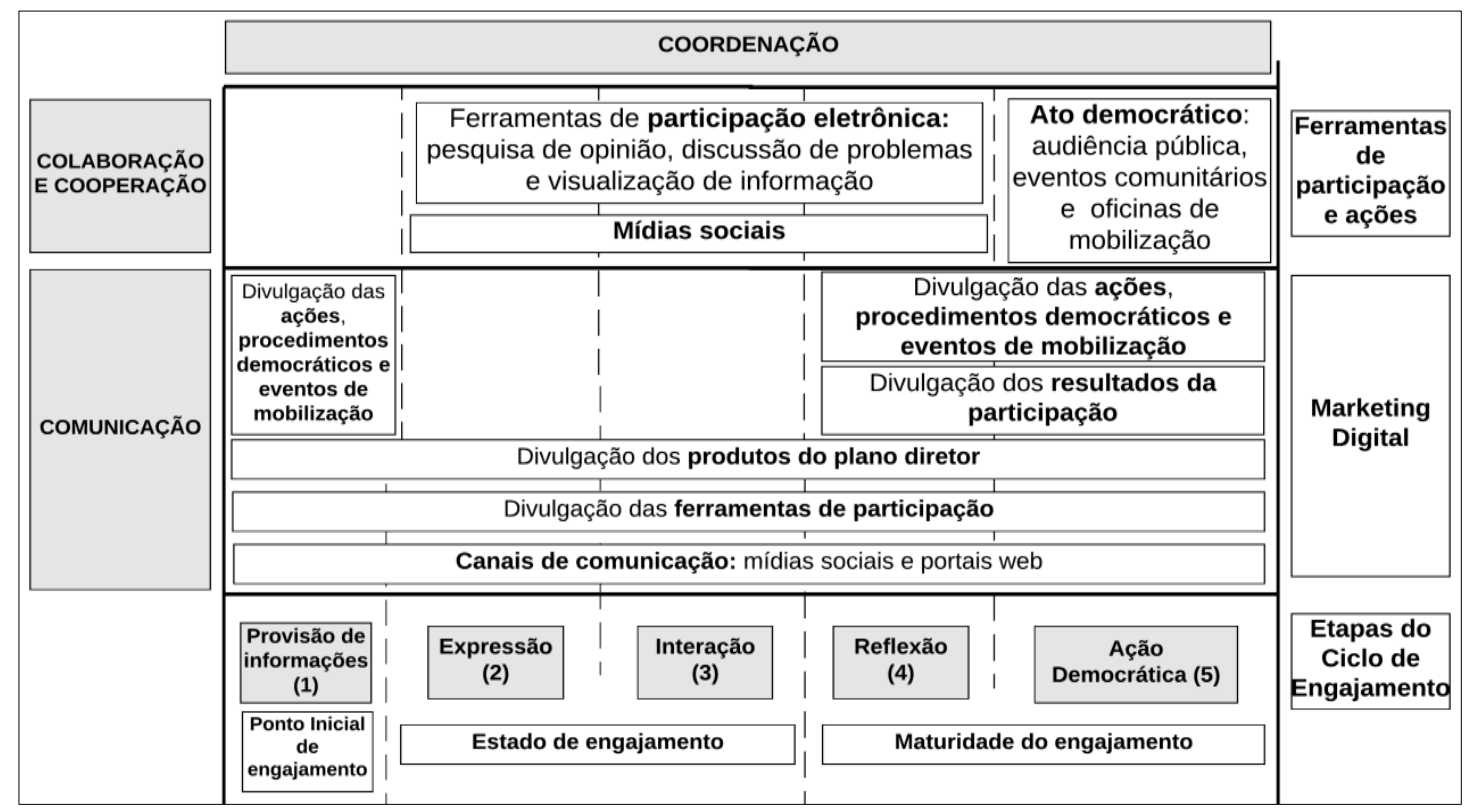

Figura 1 - Modelo de participação social do NEIRU

O fluxo de atividades é coordenado em 5 etapas: provisão de informações (1), na qual a gestão estimula o cidadão a participar por meio de divulgação das ações, procedimentos democráticos, eventos de mobilização e ferramentas de participação disponíveis; expressão (2), etapa na qual deve ser promovida a coleta de opinião do cidadão com uso de ferramentas de participação eletrônica; interação (3), na qual, os resultados e contribuições devem ser discutidos; reflexão (4), em que o cidadão reflete sobre sua experiência por meio de visualização das informações sobre os resultados parciais ou finais das ações e suas contribuições; por fim, (5) etapa na qual as ações democráticas culminam com a participação efetiva do cidadão nos eventos de mobilização, como audiências públicas, oficinas de mobilização e eventos comunitários.

\subsection{Cooperação e Colaboração}

No processo de revisão de planos diretores, os produtos parciais que deverão compor as diretrizes e fundamentos dos planos precisam ser construídos de forma colaborativa entre os cidadãos e gestores por meio dos eventos de mobilização. Além das audiências públicas, podem ser realizados outros tipos de eventos, como as oficinas comunitárias. Além disso, para ampliar as possibilidades de participação, podem ser adotadas ferramentas de participação eletrônica. É importante destacar que, atualmente, devido ao aspecto legal, o evento que confere a legitimidade dos artefatos elaborados é a audiência pública. Contudo, é permitido o uso de outros mecanismos que, de alguma maneira, ou estimulem o cidadão a participar das audiências ou permitam que a opinião da sociedade seja devidamente conhecida.

Nesse estudo, as ferramentas utilizadas são o SOPA e Opina Aí. O SOPA tem como objetivo permitir que o cidadão faça discussão de problemas da sociedade e as soluções para esses problemas, utilizando por base tecnológica, as redes sociais e visualização de informação [Caetano, 2018]. Para isso, o usuário pode inserir uma questão para que seja iniciada uma discussão na qual outros cidadãos podem contribuir 
propondo soluções. Também é possível a criação de grupos para facilitar o direcionamento das questões para usuários específicos e interessados em assuntos da comunidade. A gestão pode se beneficiar da ferramenta ao requisitar opiniões públicas, divulgar eventos e utilizar a ferramenta como canal de comunicação.

A ferramenta Opina Aí é um aplicativo para dispositivos móveis que tem como objetivo coletar opinião dos cidadãos para realizar levantamento de dados. Um exemplo de uso das ferramentas se da pela revisão do plano diretor de uma cidade de pequeno porte, na qual agentes de saúde utilizam a ferramenta Opina aí para coletar informações de residentes de bairros de zona rural e urbana. O SOPA por sua vez, pode ser usado para discutir os eventos de mobilização que serão realizados para participação social.

\subsection{Comunicação}

A comunicação ocorre durante todas as etapas apresentadas na Figura 1. Para realização da comunicação, o primeiro passo é elaborar o plano de comunicação na primeira fase do plano diretor, em que são definidos os principais setores e atores da localidade. A setorização é feita unindo localidades que possuem características culturais homogêneas, que de certa forma, mantém um relacionamento no seu fluxo de pessoas. Por exemplo, em uma cidade pequena podem ser identificados dois setores, zona rural e urbana. Em relação aos atores, devem ser identificados os grupos da sociedade que possuem interesses e necessidades em comuns e serão influenciados pelos resultados do plano diretor. Por exemplo, em uma cidade cujo a atividade econômica é a produção de café, os atores podem ser a associação comercial dos agricultores e o sindicato dos trabalhadores das lavouras. Toda a estratégia de comunicação deve levar em consideração as especificidades desses atores/setores.

Nesse trabalho, a base do elemento de comunicação é o marketing digital, embora sejam sido utilizados outros canais como radio, email, aplicativos de mensagem e divulgação de material impresso. São realizadas também, reuniões presenciais entre os núcleos gestores e grupos de trabalho. As campanhas de marketing digital tem como foco a divulgação das ações tomadas pela gestão, os eventos de mobilização, os resultados obtidos em cada fase e as ferramentas de participação disponíveis. Como canal de comunicação, estão sendo utilizados portais web e mídias sociais como Instagram, Facebook e Linkedin. Para definição do método de comunicação, foi definida uma persona do NEIRU, como forma de apresentar um perfil comunicativo. O persona foi descrito como um personagem informativo, acessível, líder, amigável, sonhador, transformador, motivador, entre outros. Cada característica desse perfil serve para orientar o trabalho de criação de conteúdo para as postagens e fazer interação com a comunidade web. As postagens são realizadas três vezes na semana. Para fazer o acompanhamento e controle da comunicação, são coletados dados que são utilizados como indicadores: presença nas audiências, quantidade de seguidores nas páginas das mídias sociais e grau de envolvimento do público com as postagens. Os indicadores são analisados com a elaboração de relatórios periódicos.

Nas publicações, o conteúdo é composto por textos, imagens e vídeos e é separado pelo método 80/20 do Principio de Pareto [Sanders, 1987]. Dessa forma, 20\% do conteúdo são destinados para: chamada de audiências públicas, apresentação e valorização do NEIRU e divulgação das ferramentas de participação. Os $80 \%$ do conteúdo restante são direcionados para: curiosidades culturais, históricas e econômicas; conscientização sobre participação; conscientização socioambiental; informações sobre 
legislação, como o Estatuto da Cidade; apresentação dos instrumentos dos Planos Diretores; e notícias esporádicas e aleatórias.

\section{Conclusão}

A participação da sociedade civil, as estratégias de organização da gestão pública, e o uso de meios e tecnologias de comunicação são conceitos que podem ser aplicados ao contexto de sistemas colaborativos. O objetivo deste estudo foi apresentar uma proposta de aplicação do modelo de colaboração 4C para definir uma estratégia de participação social no cenário de revisão de planos diretores municipais. Considerando o modelo $4 \mathrm{C}$, este estudo apresentou as atividades de coordenação estruturadas em fluxo. Para a comunicação, foram consideradas estratégias de marketing digital com foco em divulgar as ações que estão sendo tomadas pela gestão, os eventos de mobilização, os resultados obtidos e as ferramentas disponíveis. E por fim, a colaboração e cooperação tiveram como base o uso de ferramentas de participação eletrônica.

Este estudo esta em andamento, e tem como limitação não ter sido realizada a validação do modelo apresentado. Sendo assim, ainda é necessário conhecer o impacto que o uso desse modelo pode causar no engajamento. Assim, é pretendida a realização de trabalhos futuros para fazer a validação do modelo. Para isso, está sendo feita uma coleta de dados durante o processo de revisão dos planos diretores como forma de avaliar o impacto que a estratégia de participação pode causar no engajamento.

\section{Referências}

Araujo, R., Cappelli, C., Diirr, B., Engiel, P., \& Tavares, R. (2011). Democracia Eletrônica. Sistemas Colaborativos., 110-121.

Brasil. (2001). Lei $n^{o}$ 10.257, de 10 de julho de 2001. Acesso em 31 de 01 de 2019, disponível em http://www.planalto.gov.br/ccivil_03/LEIS/LEIS_2001/L10257.htm

Caetano, B. P. (2018). Uma proposta para participação cidadã na solução de problemas da sociedade. Dissertação de Mestrado apresentada ao Programa de Pós-graduação em Engenharia de Sistemas e Computação, COPPE, da Universidade Federal do Rio de Janeiro.

Cortés-Cediel, M. E., Gil, O., \& Cantador, I. (2018). Defining the engagement life cycle in e-participation. 19th Annual International Conference on Digital Government Research: Governance in the Data Age. Delft, The Netherlands.

Costa, A. P., Loureiro, M. J., \& Reis, L. P. (2014). Do Modelo 3C de Colaboração ao Modelo 4C: Modelo de Análise de Processos de Desenvolvimento de Software Educativo. Revista Lusófona de Educação(27), 181-200.

O'Brien, H. L., \& Toms, E. G. (February de 2008). What is User Engagement? A Conceptual Framework for Defining User Engagement with Technology. Journal of the American Society for Information Science and Technology banner.

Sanders, R. (1987). The Pareto principle: its use and abuse. Journal of Services Marketing, 37-40. 